\title{
An Improved Anisotropic Vector Preisach Model for Nonoriented Electrical Steel Sheet Based on Iron Loss Separation Theory
}

\author{
Lixun Zhu $\mathbb{D}^{1},{ }^{1}$ Jiacheng Jiang, ${ }^{1}$ Weimin Wu, ${ }^{1}$ Wei Li $\left(\mathbb{D},{ }^{2}\right.$ Kaiyuan $\mathrm{Lu},{ }^{1,3}$ \\ and Chang-seop Koh ${ }^{4}$ \\ ${ }^{1}$ Department of Logistics Engineering, Shanghai Maritime University, Shanghai 201306, China \\ ${ }^{2}$ Department of Electrical Engineering, Tongji University, Shanghai 200092, China \\ ${ }^{3}$ Department of Energy Technology, Aalborg University, Aalborg 9220, Denmark \\ ${ }^{4}$ Department of Electrical and Computer Engineering, Chungbuk National University, Cheongju 28644, Republic of Korea
}

Correspondence should be addressed to Lixun Zhu; lixzhu@shmtu.edu.cn and Wei Li; liweimail@tongji.edu.cn

Received 15 August 2020; Accepted 15 September 2020; Published 28 September 2020

Academic Editor: Arkadiusz Zak

Copyright ( $\odot 2020$ Lixun Zhu et al. This is an open access article distributed under the Creative Commons Attribution License, which permits unrestricted use, distribution, and reproduction in any medium, provided the original work is properly cited.

An improved anisotropic vector Preisach model is proposed in this paper to describe the hysteresis properties of nonoriented (NO) electrical steel sheet (ESS) under $50 \mathrm{~Hz}$ rotating magnetic fields. The proposed model consists of three components, static hysteresis component, eddy current component, and excess component, which is based on the iron loss separation theory. The static hysteresis component is constructed by the static vector Preisach model. The proposed model is identified by the measured hysteresis properties under $1 \mathrm{~Hz}$ and $50 \mathrm{~Hz}$ magnetic fields. Finally, the experimental results prove the effectiveness of the proposed anisotropic vector hysteresis model.

\section{Introduction}

With the rapid development of the electrical motor drive technology, different types of electrical motors are designed to meet various needs. And, more and more attention has been paid to the performance and efficiency of the motor. However, to improve the performance and the efficiency of the electrical motor in the simulation design stage, accurate magnetic field distribution and iron loss analysis are extremely significant. Until now, finite element analyses (FEA) is one of the most popular and accuracy numerical methods to calculate the magnetic field distribution and the iron loss of electrical motor. However, accuracy and speed describe the hysteresis properties between magnetic field strength $(H)$ and magnetic flux density $(B)$ is quite essential to calculation accuracy and speed of FEA.

On the one hand, the magnet filed in the teeth of the motor is alternating field, and the magnet filed in the yoke is rotating filed. According to the experimental experience, the hysteresis properties under rotating field are quite different from that under alternating fields. However, until now, most of the commercial FEA software only can consider the alternating fields. Thus, the calculation accuracy is not satisfied, and it cannot be used for motor design and optimization directly. In additional, the nonoriented (NO) electric steel sheet (ESS) also presents anisotropic properties under both alternating and rotating magnetic fields in practice. Therefore, an anisotropic vector hysteresis model should be developed to describe the vector hysteresis property of ESS and combined with FEA to analyse the performance and efficiency of the motor.

Until now, to describe the vector hysteresis property of ESS and improve the accuracy of FEA, many versions of hysteresis models have been developed, such as vector Preisach model, vector Jiles-Atherton (JA) model, vector E\&S model, and vector Play models [1-4]. The Preisach model has been widely concerned by many researchers because of its perfect modelling results, and the Preisach model is constructed from the physical point of view [5]. The classical Preisach model is proposed by Preisach firstly [6], and it is applied to describe the hysteresis property by many researchers [5, 7-11]. The original vector version of the 
Preisach model is developed by using the superposition of the classical scalar Preisach models along different azimuthal direction [12-14]. And, because the anisotropic properties cannot consider in the original vector Preisach model, it is an isotropic model. To apply the vector Preisach model to FEA and analysis the iron loss, a few frequencies dependent vector Preisach models have been developed based on the iron loss separation theory [15-17]. However, the above models are limited pay attention to the anisotropic property. Therefore, some versions of the vector Preisach model which can consider the anisotropic properties have been proposed for NO ESS. However, the model results only can present weakly anisotropic property, and they do not match the experimentally measured data well.

In this paper, an improved anisotropic vector Preisach model is proposed to describe hysteresis behaviour of NO ESS, which has low anisotropic property. The proposed model consists of three components, static hysteresis component, eddy current component, and excess component, which is based on the iron loss separation. The static hysteresis component is developed by the vector Preisach model which is identified by using the experimental data from static magnetic field. The average static scalar property is measured from the ring-type core under $1 \mathrm{~Hz}$ magnetic field. And, the parameter in the proposed model is identified by using the purely rotating magnetic fields under $50 \mathrm{~Hz}$ magnetic fields whose magnitude is limited to $1.6 \mathrm{~T}$. The identification and validation of the model are performed by using measurement data of the NO ESS, 35PN440, which is obtained from a two-dimensional (2D) single sheet tester (SST).

\section{Static Preisach Hysteresis Model}

2.1. Measurement System for the Scalar Preisach Model. The vector static Preisach model consisted of the scalar static Preisach model. Therefore, the scalar version should be constructed firstly. To construct the scalar Preisach model, the static scalar hysteresis behaviour should be measured. However, the pure static hysteresis behaviour of ESS without any eddy current and excess effects is quite difficult to measure. Therefore, in this paper, the hysteresis behaviour under $1 \mathrm{~Hz}$ exciting current is considered as the static hysteresis behaviour, and the corresponding measurement system is developed shown in Figure 1. In Figure 1, the exciting coil and the B-coil are wound around the ring-type core. The exciting coil is used to generate the magnetic fields, and the $\mathrm{H}$-waveform can be obtained from the current of the exciting coil. And, there are 40 and 600 turns of the exciting coil for lower and higher $\mathrm{H}$ value measurement, respectively. The $\mathrm{B}$-coil of 20 turns is used to measure the B-waveform. The ring-type core is the lamination core, which is made up of the toroidal NO ESS,35PN440, and it is used to obtain the average scalar $B-H$ property of the specimen [18]. The inner and outer diameters of the toroidal ESS are $40 \mathrm{~mm}$ and $50 \mathrm{~mm}$, respectively. The hysteresis minor loops with different maximum value of $B$ under $1 \mathrm{~Hz}$ alternating magnetic fields can be measured by this experimental device, and the measured results can be used to identify the static Preisach model.

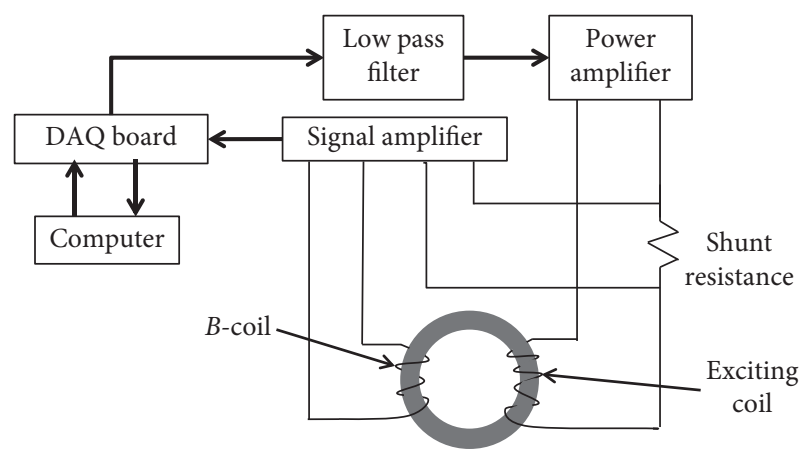

FIGURE 1: Ring-type hysteresis measurement system.

2.2. Scalar Preisach Model and Its Identification. In FEA method, the $B$-waveform is required to calculate the $H$ waveform. Therefore, an inverse version of the scalar Preisach model, which outputs $H$-waveform, can be calculated from the input $B$-swaveform by a double integral defined over the Preisach triangle [4] as follows:

$$
H=\Gamma(B)=\iint_{\alpha \geq \beta} \mu(\alpha, \beta) \gamma_{\alpha \beta}(B(t)) \mathrm{d} \alpha \mathrm{d} \beta,
$$

where $\gamma_{\alpha \beta}$ is a hysteresis operator [4], which is similar with a relay as shown in Figure 2 and given as (2), controlled by the increasing $\alpha$ and decreasing $\beta$ values of the input $B$ and can only have +1 and -1 values, $\mu$ is the distribution function of the hysteresis operators, and it should be identified by the experimental data:

$$
\gamma_{\alpha \beta}\left(B\left(t_{k}\right)\right)= \begin{cases}+1, & \text { if } B\left(t_{k}\right)>\alpha, \\ -1, & \text { if } B\left(t_{k}\right)<\beta, \\ \gamma_{\alpha \beta}\left(B\left(t_{k-1}\right)\right), & \text { if } \beta \leq B\left(t_{k}\right) \leq \alpha .\end{cases}
$$

The double integral operation in (1) will consume a lot of time when the model is applied to FEM. To improve the identification accuracy and the calculation efficiency of the Preisach model, an Everett function is defined as follows:

$$
E\left(\alpha_{0}, \beta_{0}\right)=\iint_{\alpha_{0}<B_{m}, \beta_{0}>-B_{m}} \mu(\alpha, \beta) \mathrm{d} \alpha \mathrm{d} \beta,
$$

where $\left(\alpha_{0}, \beta_{0}\right)$ is the coordinate of a point within the integral zone and $B_{m}$ is the maximum value of $B$.

Therefore, equation (1) can be instead by addition and subtraction of the Everett function as follows:

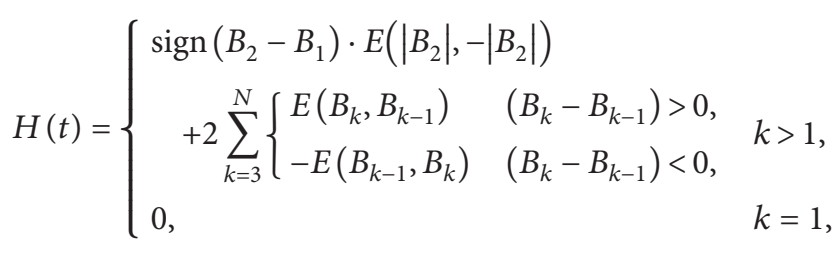

where $B_{k}$ are the extreme points of the input B-waveform, and they are stored in the extreme point memory as shown in Figure 3 [7].

In the traditional identification method, the distribution function $\mu$ or the Everett function should be identified by 


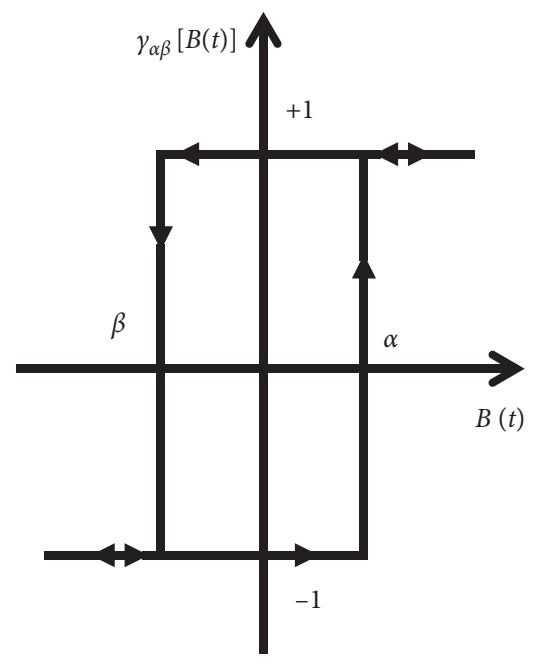

Figure 2: A hysteresis operator with the increasing value $\alpha$ and decreasing value $\beta$.

using the first-order reverse curve (FORC) [7]. However, the FORC is quite difficult to measure, and the accuracy of the measurement results is not satisfied. And, negative values will appear in the distribution function because of the measurement errors.

In this paper, the Everett function is defined from experimentally measured symmetric minor $B-H$ loops as follows:

$$
E(\alpha, \beta)= \begin{cases}h^{-}(\alpha, \alpha)-h^{-}(\alpha, \beta), & (\alpha+\beta \geq 0), \\ h^{+}(|\beta|, \alpha)-h^{+}(|\beta|, \beta), & (\alpha+\beta \leq 0),\end{cases}
$$

where $h^{+}\left(B_{a}, B\right)$ and $h^{-}\left(B_{a}, B\right)$ are, respectively, the ascending and descending branches of the symmetric $B-H$ loop with maximum amplitude $B_{a}$.

In this paper, totally 16 symmetric minor $B-H$ loops are measured from the measurement system in Figure 1, and the range of $B_{a}$ is from $0.1 \mathrm{~T}$ to $1.6 \mathrm{~T}$; the step is $0.1 \mathrm{~T}$. And, then the Everett function is identified by the measurement data and (5). The result of the Everett function which is identified by (5) is shown in Figure 4(a), and Figure 4(b) shows the comparison results between the modelling and measurement results under alternating $1 \mathrm{~Hz}$ magnet fields with different values of $B_{a}$. From the results, the modelling results can match well with the measured ones.

2.3. Vector Preisach Model. The traditional vector Preisach model consists of the superposition of classical scalar Preisach models along different azimuthal directions as follows:

$$
\begin{gathered}
\mathbf{H}=\int_{-(\pi / 2)}^{(\pi / 2)} \mathbf{e}_{\phi_{i}} H_{\varphi_{i}}\left(B_{\varphi_{i}}\right) \mathrm{d} \varphi, \\
H_{\varphi_{i}}\left(B_{\varphi_{i}}\right)=\iint_{\alpha \geq \beta} \eta\left(\alpha^{\prime}, \beta^{\prime}\right) \widehat{\gamma}\left(B_{\varphi_{i}}\right) \mathrm{d} \alpha^{\prime} \mathrm{d} \beta^{\prime},
\end{gathered}
$$

where $H_{\varphi i}$ is the output $H$-waveform of the classical scalar Preisach model along the direction of $\varphi_{i}$, having the input $B$ waveform along the direction of $B_{\varphi i}$ as input, and $\eta$ is the vector distribution function corresponding to the vector Preisach model.

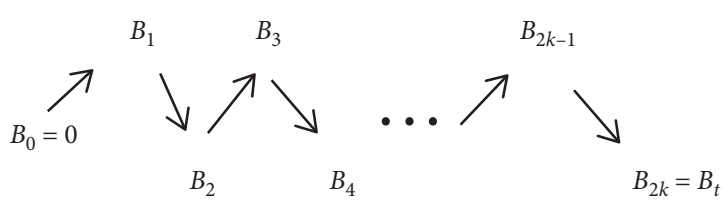

Figure 3: Extreme point memory of the input $B$-waveform.

According to the scalar Everett function $E(\alpha, \beta)$, the vector Everett function $F\left(\alpha^{\prime}, \beta^{\prime}\right)$ is proposed to implement the vector Preisach model, and it can be calculated from the classical scalar Everett function as follows:

$$
F(\alpha, \beta)=\int_{-(\pi / 2)}^{(\pi / 2)} \cos \varphi E(\alpha \cos \varphi, \beta \cos \varphi) \mathrm{d} \varphi
$$

To implement the improved anisotropic vector Preisach model, equation (5) should be rewritten to its discrete format as

$$
\mathbf{H}=\sum_{\mathrm{i}=1}^{n} \mathbf{e}_{\varphi_{i}} H_{\varphi_{i}}\left(B_{\varphi_{i}}\right) \omega_{i}
$$

where $\omega_{i}$ is the Gauss integral weight, and (6a) and (6b) can be solved by using the method which is explained in [7].

To consider the anisotropic property, the input B-waveform along the azimuthal direction $\varphi_{i}$ can be calculated as

$$
B_{\varphi_{i}}=|\mathbf{B}| \delta\left|\cos \left(\theta_{B}-\varphi_{i}+\psi\right)\right|^{1 / \omega},
$$

where $\delta=\operatorname{sign}\left(\cos \left(\theta_{B}-\varphi_{i}+\psi\right)\right), \omega$ is a parameter to consider the anisotropic property, and $\psi$ is a parameter which is used to control the initial phase of the $H$-waveform. If the parameter $\omega=1$, the output of $H$-waveform is purely circle under the circle rotating magnetic field. And, when $\omega>1$, the result is flower shape and it can simulate weakly anisotropic behavior.

\section{Proposed Preisach Hysteresis Model}

3.1. Measurement System. In this paper, a round-type twodirectional single sheet tester (R-2D-SST), as shown in Figure 5 is developed to measure the vector hysteresis properties for NO ESS under both alternating and rotating $50 \mathrm{~Hz}$ magnetic field conditions [19-21]. Its specifications are shown in Table 1.

3.2. Traditional Vector Preisach Model. Based on the theory of iron loss separation, Dlala [10] proposed a simple hysteresis model. And, the model assumes that the total $H$ is composed of three components as follows:

$$
\begin{aligned}
\mathbf{H}_{\text {total }} & =\sum_{i=1}^{n} H_{\varphi_{i}}(t) e_{\varphi_{i}} \Delta \varphi_{i}, \\
H_{\varphi_{i}} & =H_{\text {hys }}\left(B_{\varphi_{i}}(t)\right)+\frac{\sigma d^{2}}{12} \frac{\mathrm{d} B_{\varphi_{i}}(t)}{\mathrm{d} t}+\delta_{e}\left(\frac{1}{r} \frac{\mathrm{d} B_{\varphi_{i}}(t)}{\mathrm{d} t}\right)^{(1 / p)},
\end{aligned}
$$

where hysteresis is calculated from scalar Preisach model, $B_{\varphi i}$ is calculated by (9), $d$ is the thickness of ESS, $\sigma$ is the conductivity, and the parameters $r, p, \psi$, and $\omega$ can be fitted 


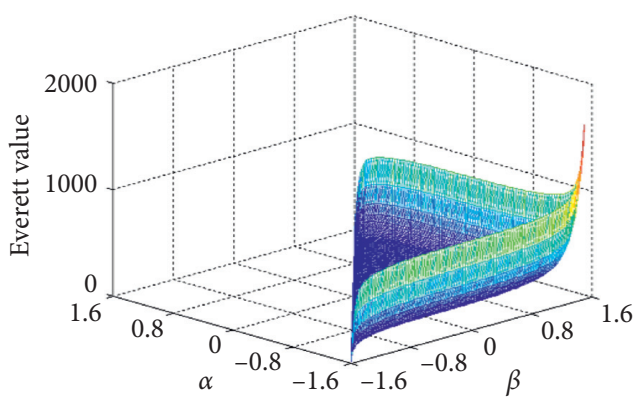

(a)

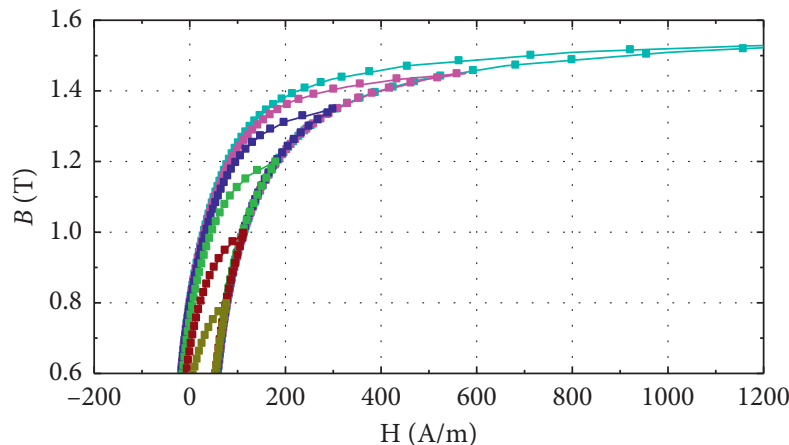

(b)

Figure 4: Modeling results of the scalar Preisach model. (a) Everett function. (b) Comparison of the measured (dot line) and modeled minor $B-H$ (solid line) loops under alternating $1 \mathrm{~Hz}$ magnetic fields.

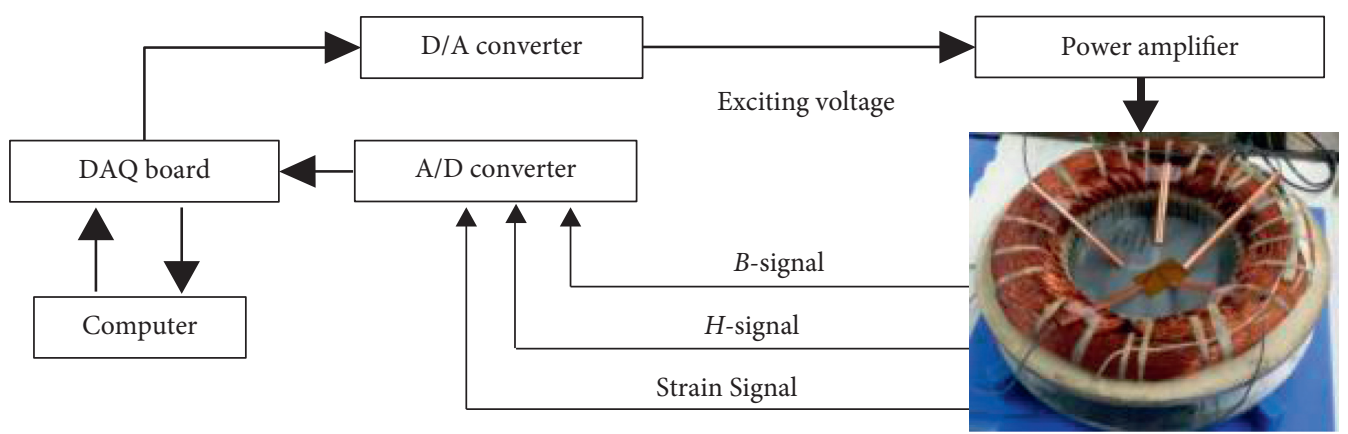

(a)

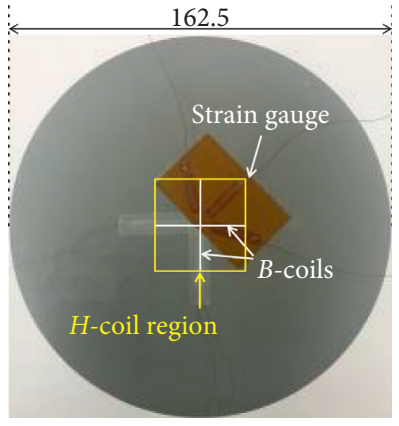

(b)

FIgURE 5: R-2D-SST for vector hysteresis measurement. (a) Overall configuration. (b) Detailed positions of the $B$-coil and $H$-coil.

TABLE 1: Specifications of the R-2D-SST system.

\begin{tabular}{lc}
\hline Item & Detail \\
\hline Type & Round \\
Specimen & Diameter $d=162.5 \mathrm{~mm}$ \\
B-coil width & $40 \mathrm{~mm}$ \\
H-coil region & $40 \times 40 \mathrm{~mm}^{2}$ \\
Shield & Upper and lower \\
\hline
\end{tabular}

by experiential data. However, the model only can describe the weakly anisotropic behavior.

3.3. Improved Model. The shape of the $H$-waveforms under the circle rotating magnetic fields with the different maximum values of $|B| B_{\max }$ is quite different. Therefore, only one set of parameters in the original model cannot match most of the rotating magnet fields. Therefore, an improved model is proposed as follows:

$$
\begin{aligned}
\mathbf{H}_{\text {total }}= & \sum_{i=1}^{n} H_{\varphi_{i}}(t) e_{\varphi_{i}} \omega_{i}, \\
H_{\varphi_{i}}= & H_{\text {hys }}\left(B_{\varphi_{i}}(t)\right) z_{i}\left(|\mathbf{B}|, \varphi_{i}\right)+\frac{\sigma d^{2}}{12} \frac{\mathrm{d} B_{\varphi_{i}}(t)}{\mathrm{d} t} \\
& +\delta_{e}\left(\frac{1}{r(B)} \frac{\mathrm{d} B_{\varphi_{i}}(t)}{\mathrm{d} t}\right)^{(1 / p(B))}, \\
B_{\varphi_{i}}= & |\mathbf{B}| \delta\left|\cos \left(\theta_{B}-\varphi_{i}+\psi(B)\right)\right|,
\end{aligned}
$$




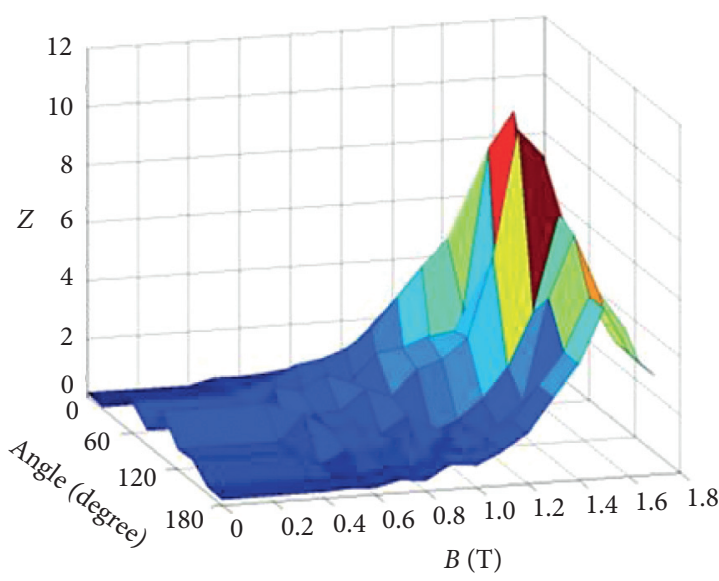

(a)

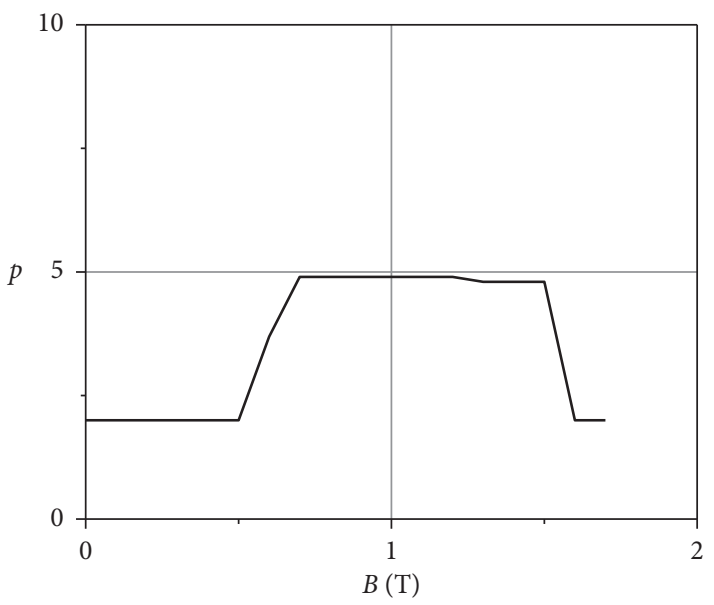

(c)

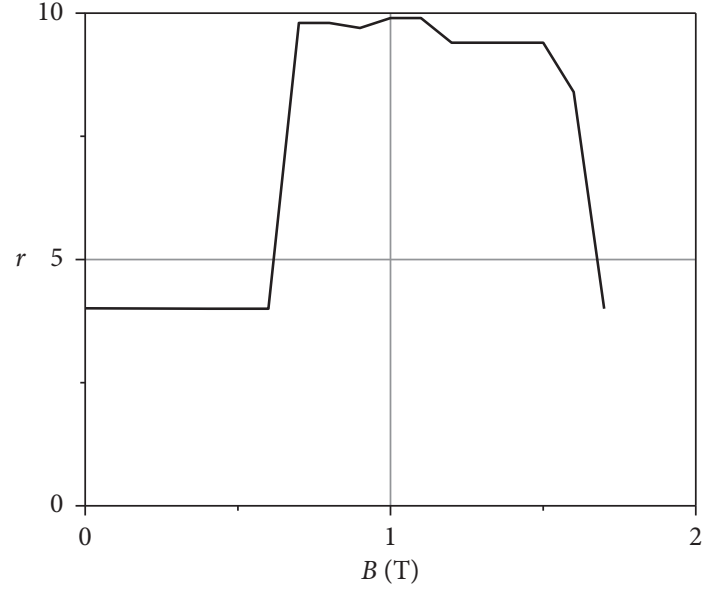

(b)

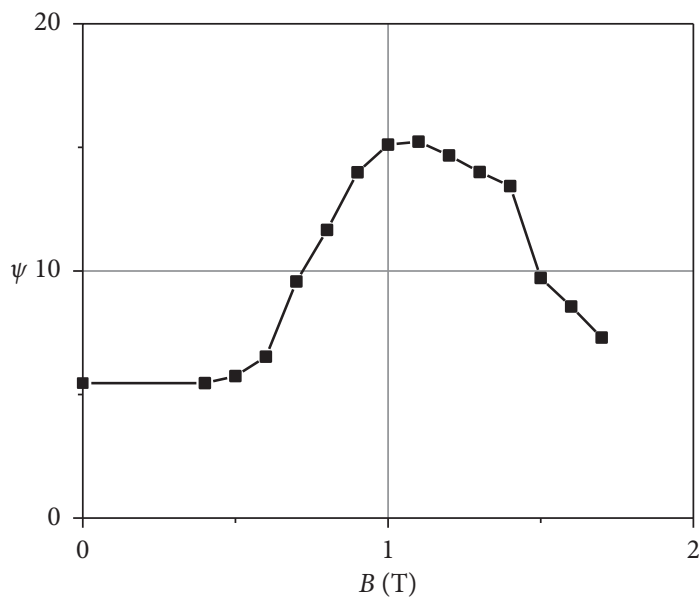

(d)

Figure 6: Identification results of parameters (a) $z$, (b) $r$, (c) $p$, and (d) $\psi$.

where $z_{i}$ is used to describe the anisotropic properties of ESS. In the model, the parameters $r, p$, and $\psi$ are assumed as piecewise linear shape functions as

$$
\begin{aligned}
& r\left(B_{j}\right)=r\left(B_{j-1}\right)+\frac{\mu_{r, j}}{\Delta B}, \\
& p\left(B_{j}\right)=p\left(B_{j-1}\right)+\frac{\mu_{p, j}}{\Delta B}, \\
& \psi\left(B_{j}\right)=\psi\left(B_{j-1}\right)+\frac{\mu_{\psi, j}}{\Delta B} .
\end{aligned}
$$

And, the parameter $z$ is assumed as a 2-D piecewise linear shape functions as

$$
z\left(\varphi_{i}, B_{j}\right)=z\left(\varphi_{i-1}, B_{j}\right)+\frac{\lambda_{z, i}}{\Delta \varphi}
$$

where $\Delta B=0.1 \mathrm{~T}$ and $B_{j}=j \Delta B, j=4,5, \ldots, 16 . \Delta \varphi=20^{\circ}$ and $\varphi_{i}=i \Delta \varphi, i=0,1, \ldots, 8$. And, $\mu_{\psi, j}, \mu_{r, j}, \mu_{p, j}$, and $\lambda_{z, i}$ can be identified from the measured vector $B$ - $H$ locus under circle rotating fields whose magnitude is $B_{j}$ by using the particle swarm optimization (PSO) method.
The objective function is defined as the root-meansquared error between the measured values and the calculated values of one $B-H$ loop under circle rotating fields whose magnitude is $B_{j}$, as follows:

$$
\begin{aligned}
\text { error } H & =\frac{1}{T} \int_{T}\left(H_{\text {exper }}-H_{\text {model }}\right)^{2} \mathrm{~d} t \\
& \approx \sqrt{\frac{1}{n} \sum_{1}^{n}\left(H_{\text {exper }}-H_{\text {model }}\right)^{2},}
\end{aligned}
$$

where $n$ is the number of sampling points of the vector hysteresis locus, $H_{\text {exper }}$ is the measure magnetic field strength values on the sampling points, and $H_{\text {model }}$ is the modelling magnetic field strength values on the sampling points.

In addition, the effective applied range of the proposed model depends on the maximum $B$ value of the measurement $B-H$ locus which is used to identify the model.

3.4. Modeling Results. In this paper, a set of vector $\mathbf{B}-\mathbf{H}$ locus of NO ESS 35PN440 under $50 \mathrm{~Hz}$ is measured by using R-2D-SST. And, the B-H locus under the pure rotating 


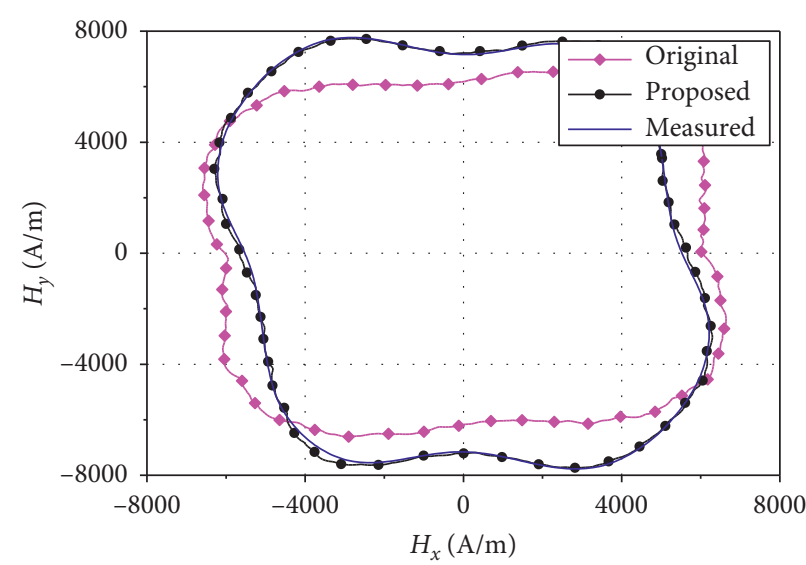

(a)

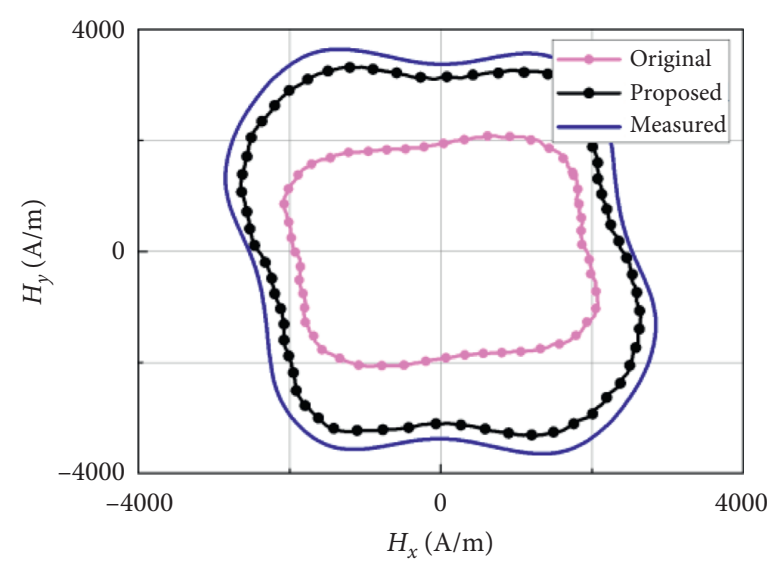

(b)

Figure 7: Comparison of the measured, original, and proposed results under circle magnetic fields. (a) $B_{\max }=1.6 \mathrm{~T}$. (b) $B_{\max }=1.48 \mathrm{~T}$.

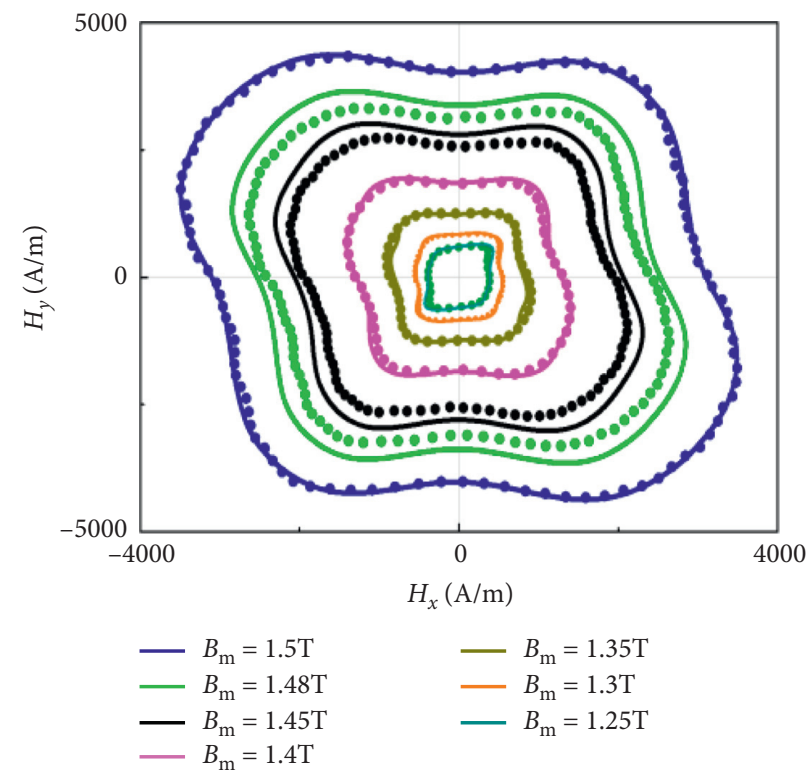

FIGURE 8: Modeling results comparison of proposed method (mark) and measured data (solid line) under circle magnetic field with different $B_{\max }$ values.

magnet field for the range of $0.4 \leq B_{\max } \leq 1.6$ (T), which step is $0.1 \mathrm{~T}$, is used to identify the parameters in the proposed vector Preisach model by using PSO method. The identification results of parameter $z, r, p$, and $\psi$ are shown in Figure 6.

The measured, original, and proposed modelling $\mathbf{H}$ locus under circular rotating magnetic field conditions when $B_{\max }=1.6 \mathrm{~T}$ and $B_{\max }=1.48 \mathrm{~T}$ are compared and shown in Figure 7. From the figure, the accuracy of the proposed model is much higher than the original model under the both rotating magnetic fields. In addition, the measured $\mathbf{H}$ locus when $B_{\max }=1.6 \mathrm{~T}$ is used to identify the parameter in the proposed model, while when $B_{\max }=1.48 \mathrm{~T}$ it is not used.

The measured and improved modelling $\mathbf{H}$ locus under circular rotating magnetic field conditions with different $B_{\max }$ value is compared and shown in Figure 8. In the figure, the $\mathbf{H}$ locus for $B_{\max }=1.3 \mathrm{~T}, 1.4 \mathrm{~T}$, and $1.5 \mathrm{~T}$ is used in the identification of parameters $z, r$, $p$, and $\psi$. While those for $B_{\max }=1.25 \mathrm{~T}, 1.35 \mathrm{~T}, 1.45 \mathrm{~T}$, and $1.48 \mathrm{~T}$, it is not used. From the figure, the proposed vector hysteresis model can predict the vector hysteresis properties very accurately under various rotating magnetic fields. However, the accuracy when $B_{\max }$ is $1.45 \mathrm{~T}$ and $1.48 \mathrm{~T}$ is lower than others because of the interpretation method is used to obtain the parameter in the model.

The $\mathbf{H}$ locus predicted by the proposed model for alternating magnetic fields of $B_{m}=1.6 \mathrm{~T}$ along rolling and transverse direction is shown in Figure 9 together with that from measured data. From the figure, the proposed model also can simulate the hysteresis properties for unidirectional scalar hysteresis properties. And, the deviation $d_{H}$ between the measured and modelling results along rolling and transverse direction is $3.47 \%$ and $4.51 \%$, respectively. And, the deviation, $d_{H}$, is defined as 


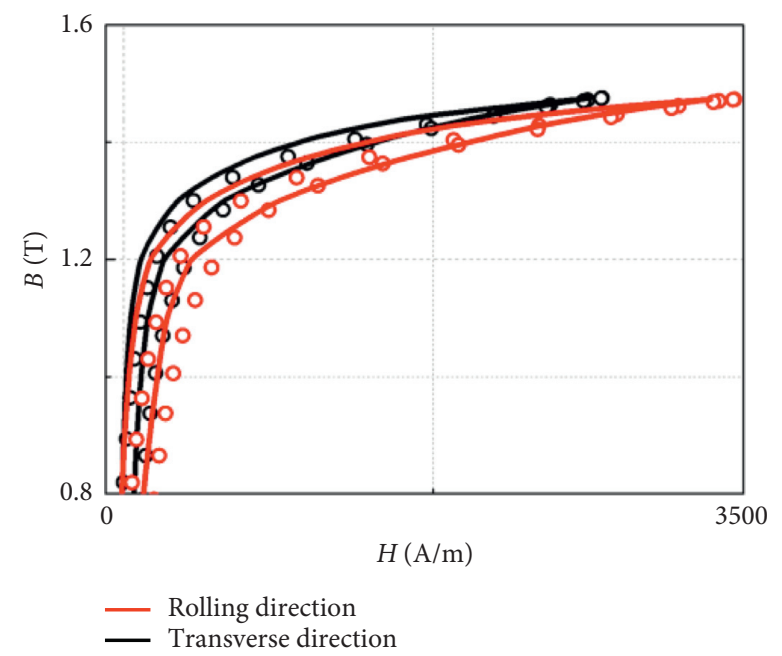

Figure 9: Result comparison of proposed model (solid line) and measured data (mark) under alternating magnetic fields along rolling and transverse directions.

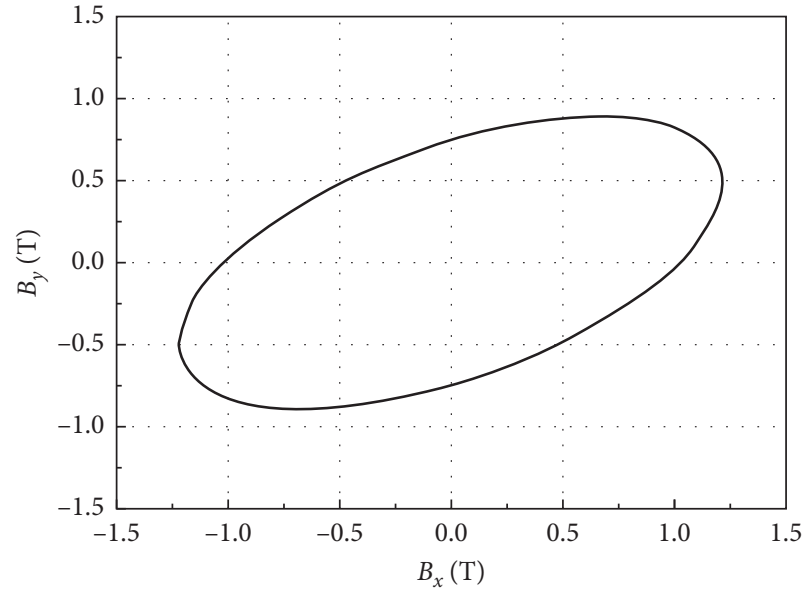

(a)

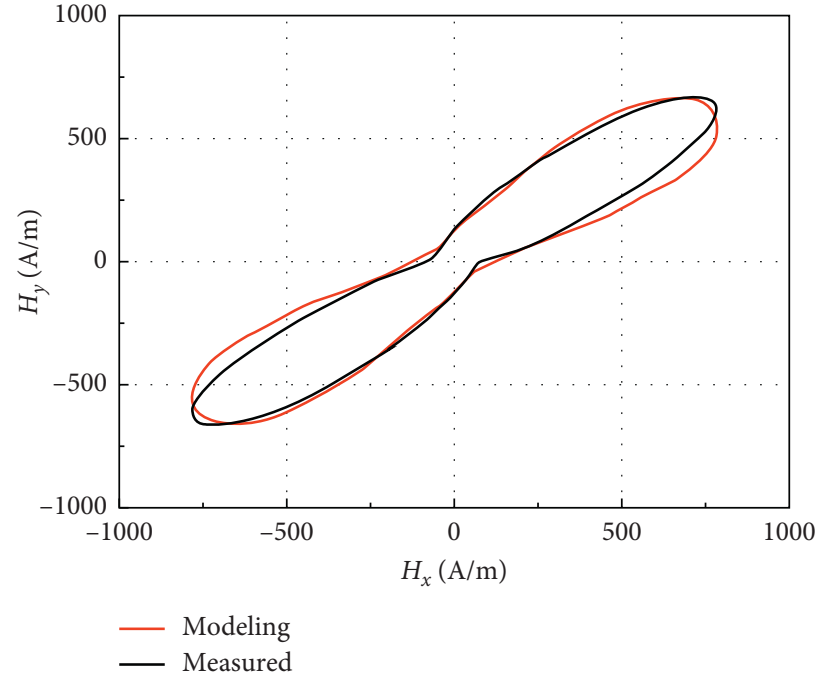

(b)

FIGURE 10: Modeling result comparison of proposed method and measured data under ellipse magnetic field. (a) The input B locus. (b) The output $H$ locus.

$$
d_{H}=\frac{\sqrt{\sum_{i=1}^{N}\left(H_{\text {mea }}\left(\tau_{i}\right)-H_{\text {mod }}\left(\tau_{i}\right)\right)^{2}}}{N\left\{\max \left(H_{\text {mea }}\right)-\min \left(H_{\text {mea }}\right)\right\}} \times 100[\%],
$$

where $N$ is the number of sampling points of the $B-H$ loop, $\tau$ stands for $\omega t \in[0,2 \pi]$ with as the angular frequency of a $B$ waveform, and $H_{\text {mea }}$ and $H_{\text {mod }}$ are the measured and modelling $H$-waveforms, respectively.

Figure 10 shows the modelling performance of the proposed vector hysteresis model when it is applied to elliptically rotating magnetic field conditions. The predicted and measured results match well each other, and it is shown that the proposed vector hysteresis model can be successfully applied to various rotating magnetic field conditions.

\section{Conclusion}

In this paper, an improved anisotropic vector Preisach hysteresis model is proposed to describe the vector and weakly anisotropic hysteresis behavior for NO ESS under $50 \mathrm{~Hz}$ rotating magnetic fields. The proposed model consists of three components, static hysteresis component, eddy current component, and excess component, and coefficients $z, r, p$, and $\psi$ are introduced to increase the anisotropic property and the accuracy. The proposed model is identified by the measured hysteresis properties under $1 \mathrm{~Hz}$ and $50 \mathrm{~Hz}$ alternating and rotating magnetic fields. And, because maximum $B$ value of the measured data for identification is $1.6 \mathrm{~T}$, the limitation of the model is only up to the magnetic field whose $B_{\max }=1.6 \mathrm{~T}$. Through applications to NO ESS, 
the proposed vector hysteresis model is proven to describe the anisotropic properties under various rotating magnetic fields.

\section{Data Availability}

The data used to support the findings of this study are available from the corresponding author upon request.

\section{Conflicts of Interest}

The authors declare no conflicts of interest.

\section{Acknowledgments}

This work was supported by the National Natural Science Foundation of China under the Grants 51777139 and 52007113 and the Shanghai Sailing Program under the Grant 20YF1416300.

\section{References}

[1] Y. Masayuki, U. Shohei, W. Daisuke, and E. Masoto, "Vector magnetic properties and two-dimensional magnetostriction of various soft magnetic materials," International Journal of Applied Electromagnetics and Mechanics, vol. 44, no. 3, pp. 387-400, 2014.

[2] E. Fallah and J. S. Moghani, "A new identification and implementation procedure for the isotropic vector Preisach model," IEEE Transactions on Magnetics, vol. 44, no. 1, pp. 37-42, 2017.

[3] L. Zhu and C. S. Koh, "A novel vector hysteresis model using anisotropic vector play model taking into account rotating magnetic fields," IEEE Transactions on Magnetics, vol. 53, no. 6, Article ID 7300604, 2017.

[4] I. D. Mayergoyz, Mathematical Models of Hysteresis and Their Applications, Elsevier, Amsterdam, Netherlands, 2003.

[5] N. Duan, W. Xu, S. Wang, J. Zhu, and Y. Guo, "Hysteresis modelling of high-temperature superconductor using simplified Preisach Model," IEEE Transactions on Magnetics, vol. 51, no. 3, Article ID 7300904, 2015.

[6] F. Preisach, "Über die magnetische nachwirkung," Zeitschrift für Physik, vol. 94, no. 5-6, pp. 277-302, 1935.

[7] I. D. Mayergoyz, Mathematical Models of Hysteresis and Their Applications, Elsevier Science Inc., New York, NY, USA, 2003.

[8] S. Hussain and D. A. Lowther, "An efficient implementation of the classical Preisach model," IEEE Transactions on Magnetics, vol. 54, no. 3, Article ID 7300204, 2018.

[9] G. Bertotti, "Dynamic generalization of the scalar Preisach model of hysteresis," IEEE Transactions on Magnetics, vol. 28, no. 5, pp. 2599-2601, 1992.

[10] M. Ruderman and T. Bertram, "Identification of soft magnetic B-H characteristics using discrete dynamic Preisach model and single measured hysteresis loop," IEEE Transactions on Magnetics, vol. 48, no. 4, pp. 1281-1284, 2012.

[11] R. Zeinali, D. C. J. Krop, and E. A. Lomonova, "Comparison of Preisach and congruency-based static hysteresis models applied to non-oriented steels," IEEE Transactions on Magnetics, vol. 56, no. 1, Article ID 6700904, 2020.

[12] L. Zhu, W. Wu, X. Xu et al., "An improved anisotropic vector Preisach hysteresis model taking account of rotating magnetic fields," IEEE Transactions on Magnetics, vol. 55, no. 6, Article ID 7300404, 2019.
[13] E. Fallah and J. S. Moghani, "A new identification and implementation procedure for the isotropic vector Preisach model," IEEE Transactions on Magnetics, vol. 44, no. 1, pp. 37-42, 2008.

[14] A. A. Adly and S. K. A.-E. Hafiz, "Efficient implementation of anisotropic vector Preisach-type models using coupled step functions," IEEE Transactions on Magnetics, vol. 43, no. 6, pp. 2962-2964, 2007.

[15] P. Handgruber, A. Stermecki, O. Bíró et al., "Anisotropic generalization of vector Preisach hysteresis models for nonoriented steels," IEEE Transactions on Magnetics, vol. 51, no. 3, Article ID 7300604, 2015.

[16] E. Dlala, "Efficient algorithms for the inclusion of the Preisach hysteresis model in nonlinear finite-element methods," IEEE Transactions on Magnetics, vol. 47, no. 2, pp. 395-408, 2011.

[17] M. Kuczmann and G. Kovács, "Improvement and application of the viscous-type frequency-dependent Preisach model," IEEE Transactions on Magnetics, vol. 50, no. 2, Article ID 7009404, 2014.

[18] J. Kitao, K. Hashimoto, Y. Takahashi et al., "Magnetic field analysis of ring core taking account of hysteretic property using play model," IEEE Transactions on Magnetics, vol. 48, no. 11, pp. 3375-3378, 2012.

[19] L. Zhu, H. S. Yoon, and C. S. Koh, "Measurement and modeling of magnetostriction characteristics of highly Grain-oriented electrical steel sheet by using round-type two-dimensional single sheet tester," IEEE Transactions on Magnetics, vol. 55, no. 1, pp. S78-S96, 2017.

[20] L. Zhu, J. Lim, and C. S. Koh, "Comparison of two-dimensional single sheet testers for the measurements of vector hysteresis and magnetostriction characteristics," IEEE Transactions on Magnetics, vol. 55, no. 1, pp. S141-S146, 2017.

[21] L. Zhu, H. S. Yoon, H. J. Cho et al., "Finite-element analysis of magnetostriction force in power transformer based on the measurement of anisotropic magnetostriction of highly grainoriented electrical steel sheet," IEEE Transactions on Magnetics, vol. 52, no. 3, Article ID 6100304, 2015. 